\title{
Spectrally Efficient Multi-Carrier Modulation Using Gabor Transform
}

\author{
Shravan Sriram, Naveenkumar Vijayakumar, P. Aditya Kumar, Akash S. Shetty, V. P. Prasshanth, \\ K. A. Narayanankutty
}

Department of Electronics and Communication Engineering, Amrita School of Engineering, Amrita Vishwa Vidyapeetham University, Coimbatore, India.

Email: sshravan91@gmail.com,naveen.vijayakumar@gmail.com,ka_narayanankutty@cb.amrita.edu

Received February $16^{\text {th }}, 2013$; revised March $15^{\text {th }}, 2013$; accepted March $23^{\text {rd }}, 2013$

Copyright (C) 2013 Shravan Sriram et al. This is an open access article distributed under the Creative Commons Attribution License, which permits unrestricted use, distribution, and reproduction in any medium, provided the original work is properly cited.

\begin{abstract}
Non Orthogonal Frequency Division Multiplexing (NOFDM) systems make use of a transmission signal set which is not restricted to orthonormal bases unlike previous OFDM systems. The usage of non-orthogonal bases generally results in a trade-off between Bit Error Rate (BER) and receiver complexity. This paper studies the use of Gabor based on designing a Spectrally Efficient Multi-Carrier Modulation Scheme. Using Gabor Transform with a specific Gaussian envelope; we derive the expected BER-SNR performance. The spectral usage of such a NOFDM system when affected by a channel that imparts Additive White Gaussian Noise (AWGN) is estimated. We compare the obtained results with an OFDM system and observe that with comparable BER performance, this system gives a better spectral usage. The effect of window length on spectral usage is also analyzed.
\end{abstract}

Keywords: Non-Orthogonal Frequency Division Multiplexing (NOFDM); Spectrally Efficient Frequency Division Multiplexing (SEFDM); Gabor Transform; OFDM; Reisz Bases; Multi Carrier Modulation (MCM)

\section{Introduction}

With a growth in the number of users, there is an increase in the demand for spectrum. Research pertaining to the efficient use the available spectrum has produced numerous results over the past few years. This work came to fruition in the form of Orthogonal Frequency Division Multiplexing, which is a special class of Multiple Carrier Modulation that has emerged as a leading candidate for high data rate wireless communication. The main advantage of this is its ease of implementation that eliminates ICI at low receiver complexity.

Ahmed et al. in [1] introduced a Spectrally Efficient Frequency Division Multiplexing (SEFDM) system which is spectrally more efficient than OFDM. In this SEFDM system non-orthogonality was introduced by a parameter $\alpha$, where $\Delta f=\alpha / T$ and $0<\alpha<1$. The above discussed system requires high frequency precision in order to reduce frequency offset effects. In addition, it will require different equipment for different values of frequency spacing. To overcome the limitations of this system a technique is discussed in $[2,3]$ where, the transmitted symbol set was modified by zero padding which leads to higher frequency resolution on performing IDFT. The partial transmission of the IDFT results in spectrum compression.

By exploiting the circular conjugate symmetry property of Discrete Fourier Transform (DFT) better spectral efficiency \& BER-SNR performance was achieved as discussed in [4]. All these systems make use of non-orthogonal bases for which an inverse doesn't exist. This results in greater receiver complexity. In this paper we develop an NOFDM system that deviates from the conventional system as discussed in [5] where $\Delta f T<1$, by introducing a non-orthogonal basis set that possess a dual, which can be used in the receiver to retrieve the transmitted symbol, hence simplifying the receiver design. In order to understand the NOFDM system, a clear understanding of the working of an OFDM system is a prerequisite.

This paper is organized as follows: Section 2 gives an insight into the critical concepts behind the working of an OFDM system that are critical for a clear understanding of an NOFDM system. Section 3 begins with a brief introduction on NOFDM, and proceeds to discuss the significance of Gabor bases and their use in the implemen- 
tation of an NOFDM system. A typical OFDM system has been considered as the baseline system. Section 4 gives the simulation results of the proposed NOFDM system in comparison with the baseline OFDM system. Conclusions are drawn from the simulation results that help in developing an NOFDM system with optimal parameters resulting in better spectral efficiency and BERSNR performance.

\section{OFDM}

In an OFDM system, the symbols obtained after constellation mapping are modulated onto orthogonal signals of different frequencies corresponding to each of the subcarriers used [6].

$$
S(n)=X_{k} * S_{k}(n) ;
$$

where, $X_{k}$ represents the symbols to be transmitted and $S_{k}(n)$ represents each subcarrier given by,

$$
S_{k}(n)=\cos 2 \pi k n / N-j \sin 2 \pi k n / N
$$

On imposing Hermitian symmetry to the symbols i.e. $X_{N-K}=X_{k}$ for a symbol stream of length $N / 2$ we end up with a stream of length $N$ where the symbols are symmetric about $X_{N / 2}$. Because of the use of orthogonal carriers, this modulation of symbols can be simplified and represented as taking an IFFT of the stream of symbols. Thus the IFFT and FFT pair performs the operation of modulation and demodulation of the symbols onto orthogonal Sine and Cosine subcarriers (depicted in Figure 1).

Another concept of interest is the use of cyclic prefix for the diagonalization of the channel matrix. It is this diagonalization that enables channel partitioning. Cyclic prefix also helps in the removal of ISI. Thus the transmitter portion of the OFDM system can be visualized as depicted in Figure 2.

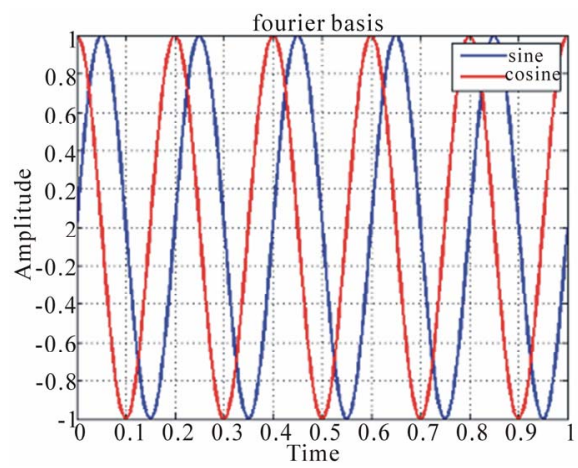

Figure 1. Fourier basis for specific channel.

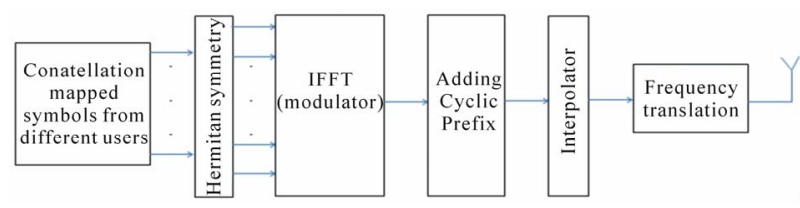

Figure 2. Transmitter of an OFDM system.

\section{NOFDM}

Non Orthogonal Frequency Division Multiplexing provides us with a major advantage of spectral efficiency, as the underlying pulse can be chosen with sharper frequency domain decay. Also the modulation scheme based on Reisz bases tends to be more robust against frequency selective fading [5]. The use of non-orthogonal basis functions (Riesz basis) is the key idea in this paper.

\subsection{Gabor Transform}

Gabor transform is a type of Short Time Fourier Transform (STFT). In STFT, the input symbols are first windowed in the time domain and then the Fourier transform operation is performed on the windowed symbol set. The windowing operation removes the orthogonality of the basis functions [7]. In Gabor transform a Gaussian window is used for windowing. It is given by,

$$
w(n)=\left(\frac{1}{2 \pi}\right)^{1 / 2} \cdot \mathrm{e}^{-\sigma n^{2} / 2}
$$

Gabor transform is a two dimensional transform in which the response depends on the frequency of the signal and the time at which windowing is performed.

The basis functions of Gabor transform are non-orthogonal which means that the basis functions are not invertible. But, a unique property of a Gabor bases is that it is that there exists a dual basis [8] set such that the original signal can be recovered by a transformation operation which uses the dual basis set.

\subsection{Implementation of NOFDM System}

In the NOFDM system the loss of orthogonality calls for the use of non-orthogonal signals for mapping the symbols onto. Hence to induce non orthogonality we make use of a form of Gabor transform for modulation. Here, we go with a combination of Gaussian and hanning window as the final combination that performs windowing in the time domain. This window is given by,

$$
w(n)=\left(\frac{1}{2}\right) \cdot\left(\frac{1}{2 \pi}\right)^{\frac{1}{2}} \cdot\left(1-\cos \left(\frac{2 \pi n}{N-1}\right)\right) \cdot \mathrm{e}^{-\frac{\sigma n^{2}}{2}}
$$

We use Hanning window in order to provide finite support for the Gaussian window. We select this window approximately eight times the $3 \sigma$ of the Gaussian window. This window and FFT combination can be approximated as performing the modified Gabor transform (since the window is not just Gaussian in nature) on the signal. This Gabor basis set (each basis corresponding to a specific carrier) obtained, used for modulating and demodulating the symbols from different users is as shown in Figure 3. 
It was observed that for $\sigma$ value of $2 / \pi$ we obtain better results while using Gabor bases in image processing [9], using this results in the Gabor Bases a minimum $3 \mathrm{~dB}$ bandwidth is observed. So, we use this value for implementation of the Modified Gabor transform in the NOFDM system. Also, it is observed that the BER-SNR performance improves as the value of sig decreases and an optimal performance is obtained at a sig value of $2 / \pi$ as shown in Figure 4.

In the NOFDM system we modulate the symbols using the Modified Gabor basis signals which follow the equation,

$$
s(n)=\sum_{k=0}^{N-1} x_{k}(n) w(n) \mathrm{e}^{-j 2 \pi f_{k} t}
$$

At the receiver, to demodulate the transmitted signal we use the Inverse Gabor transform whose bases are the conjugate of the Gabor basis [8]. The Gaussian window used in the inverse transform is given by,

$$
W^{\prime}(n)=(1 / 2 \pi)^{1 / 2} \cdot \mathrm{e}^{\frac{\sigma n^{2}}{2}}
$$

The inverse transform is given by,

$$
y(n)=\sum_{k=0}^{N-1} r(n) W^{\prime}(n) \mathrm{e}^{j 2 \pi f_{k} t}
$$

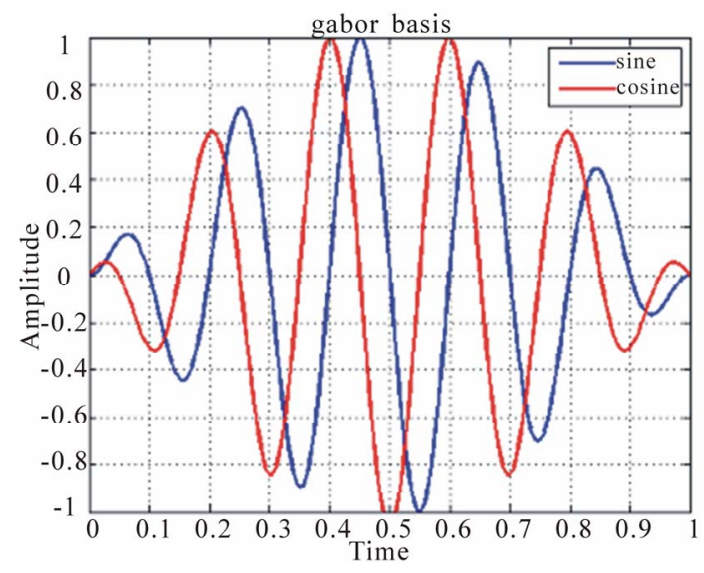

Figure 3. Gabor basis for a specific channel.

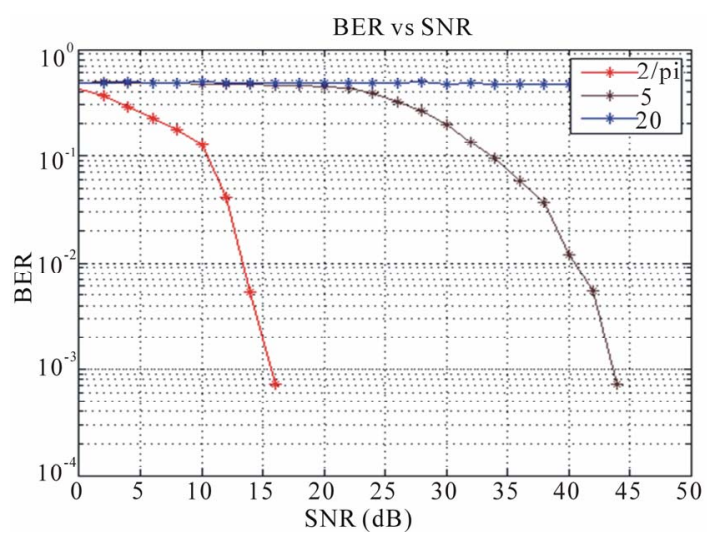

Figure 4. BER-SNR for $\sigma=2 / \pi, 5,20$.
In the NOFDM system, we define a factor " $p$ " that is a power of 2 . This " $p$ " is a ratio of the symbols to be transmitted to the window length. " $N / p$ " subcarriers are used for the transmission of $N$ symbols. Due to windowing operation (use of Gabor basis) the $N / p$ subcarriers can be used to facilitate the transmission of the $p$ sets of symbols. The Hanning window used ensures that the Gaussian window goes to zero outside the specified window length. This minimizes abrupt changes in the transmitted signal and hence leads to a much sharper frequency response. The transmitted signal in the NOFDM system is given by,

$$
\begin{aligned}
v(n) & =\sum_{a=0}^{\frac{N}{p}-1} \sum_{k=0}^{p-1} \sum_{n=1}^{N / p}\{w(n-k N / p) \\
& \left.\times X_{a}(n+k N / p) \cdot \mathrm{e}^{-j 2 \pi\left(F_{c}+a / N\right) n}\right\}
\end{aligned}
$$

where,

$$
p=N /(\text { window length })
$$

\section{Simulation Results and Conclusions}

The following results are for an OFDM and NOFDM system designed for the Digital Video Broadcasting (DVB) standard of European terrestrial digital television (DTV) service based on [10]. Our simulations will focus in the $2 \mathrm{k}$ mode of the DVB-T standard which uses 1705 carriers which carry symbols with a useful duration of $224 \mathrm{~ms}$.

From the plots (a) and (b) of Figure 5 we find that the bandwidth decreases from $8 \mathrm{MHz}$ to $4 \mathrm{MHz}$ as the factor $p$ changes from 1 to 4 . This implies that the bandwidth (B) used decreases with the window length i.e. B is proportional to $N / p$ (9). From the plots (b) and (c) of Figure 5 we observe that the bandwidth used is almost the same but there is a decrease in the power of the out of band emissions.

On transmitting the symbols on a set of subcarriers over a channel that imparts AWGN noise, one can observe that the recovery of the symbols is possible in both the OFDM and NOFDM systems and the data transmitted through the NOFDM system has a BER just slightly higher than that transmitted through an OFDM system for a given SNR (depicted in Figure 6).

It is also observed that the BER-SNR performance improves for the NOFDM system with a decrease in the window length used to develop the non-orthogonal basis set as shown in Figure 7. Since the main role of cyclic prefix is to maintain the orthogonality of the sub channels, it must be noted that no cyclic prefix is needed in the NOFDM system. Thus the NOFDM system designed does not have cyclic prefix. However, cyclic prefix can be incorporated if necessary in order to combat ISI.

Thus from the results obtained we can conclude that 

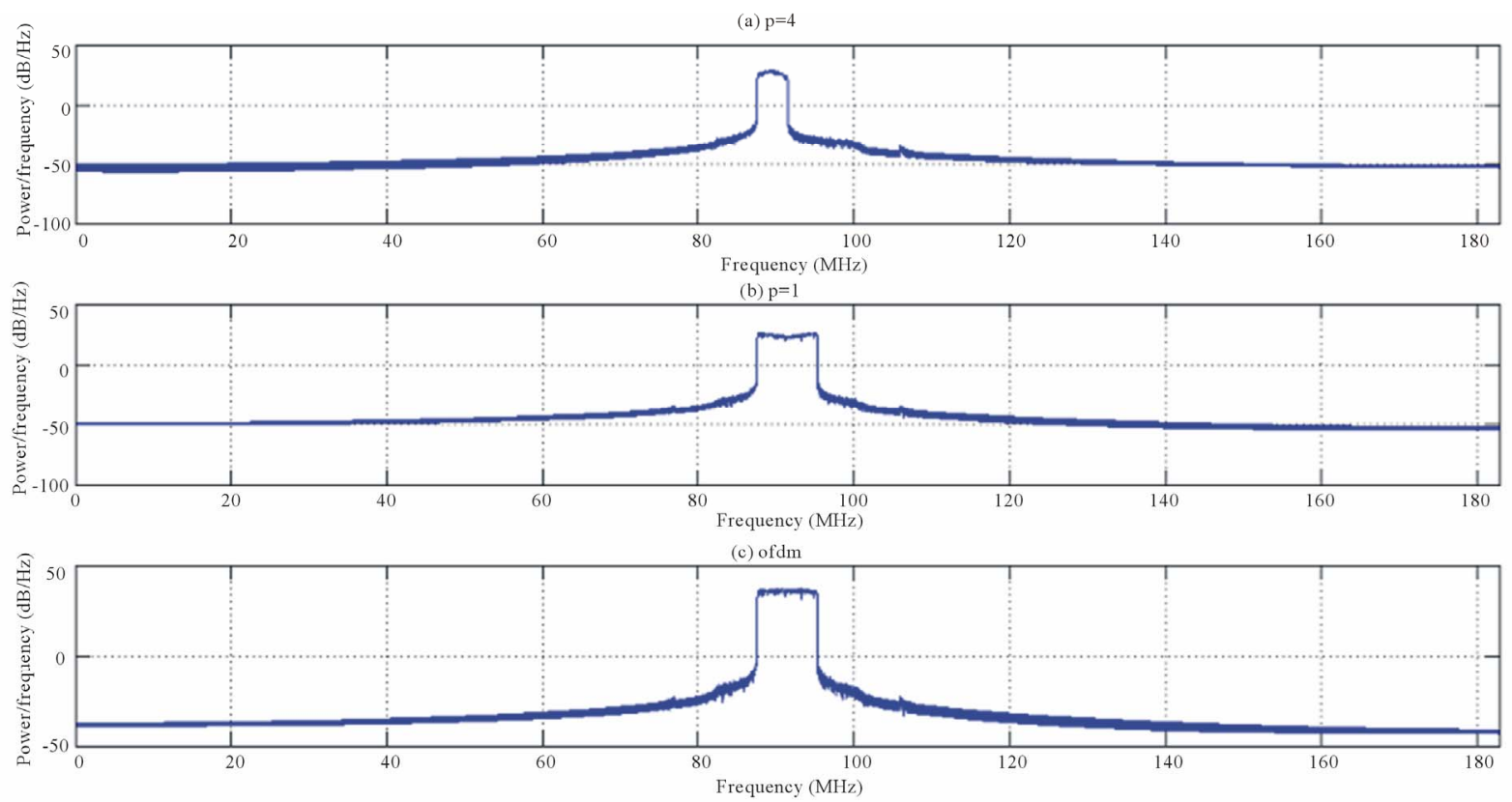

Figure 5. Power Spectral Density of the transmitted signal given $N=4096$ for (a) $p=4$ in an NOFDM system; (b) $p=1$ in an NOFDM system; (c) OFDM system.

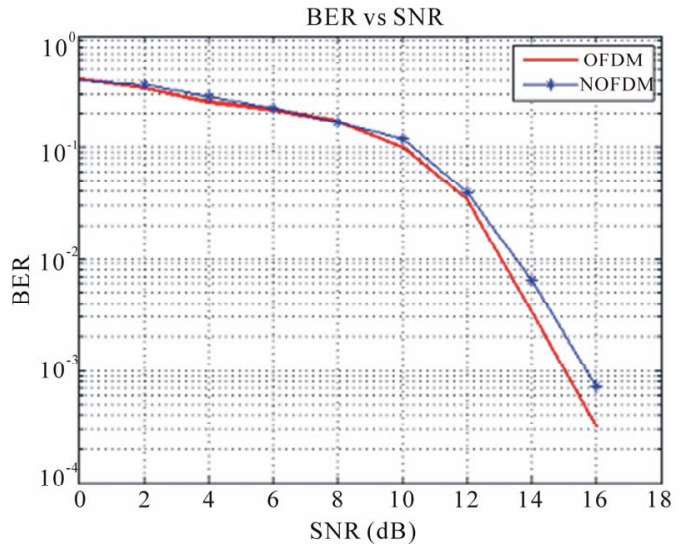

Figure 6. BER vs SNR for an OFDM and an NOFDM system.

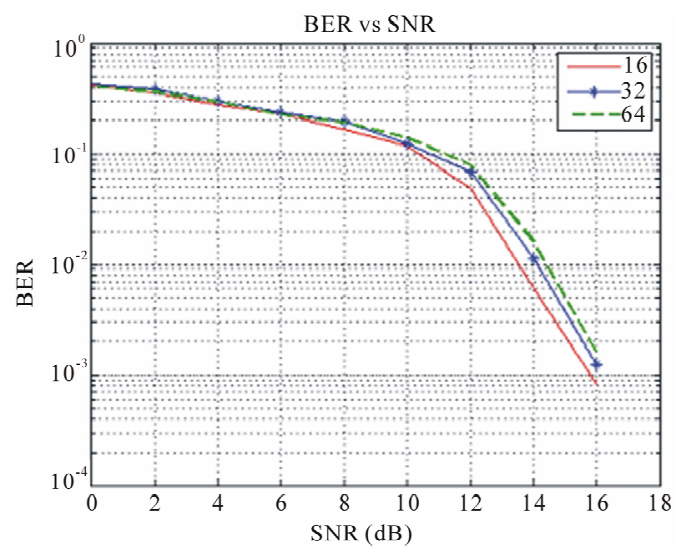

Figure 7. BER vs SNR for a NOFDM system for different window lengths. the spectrum of the NOFDM signal transmitted is occupies lesser bandwidth when compared to that of the OFDM system when $p>1$ (where, $p=1,2,4,8, \cdots$ ). This is a major advantage over the OFDM system. Since the BER-SNR performance of this system remains comparable with that of the baseline OFDM system, the developed system is an improvement over OFDM in DVB-T.

\section{Summary}

The NOFDM system has been implemented. The BER vs. SNR performance for a system implemented using a Gabor basis with sigma value of $2 / \pi$ was found to be optimal and comparable to that of an OFDM system. The spectrum is found to be efficient than that of OFDM systems and also the bandwidth is found to be inversely proportional to the window length of the Gabor transform.

\section{Acknowledgements}

We thank the faculty of the department of Electronics and Communication Engineering of Amrita Vishwa Vidyapeetham for their support and motivation.

\section{REFERENCES}

[1] M. Rodrigues and I. Darwazeh, "A Spectrally Efficient Frequency Division Multiplexing Based Communication System," 8th International OFDM Workshop, Hamburg, September 2003, pp. 70-74.

[2] S. I. A. Ahmed and I. Darwazeh, "IDFT Based Transmitters for Spectrally Efficient FDM System," London Communications Symposium, 2009. 
http://www.ee.ucl.ac.uk/lcs/previous/LCS2009/LCS/lcs09 37.pdf

[3] S. Isam and I. Darwazeh, "Simple DSP-IDFT Techniques for Generating Spectrally Efficient FDM Signals," 2010 7th International Symposium on Communication Systems Networks and Digital Signal Processing (CSNDSP), London, 21-23 July 2010, pp. 20-24.

[4] Z. Zhang, W. Chen, Q. Liu and G. Xuan, "NOFDM System Based on Circular Conjugate Symmetry Properties of DFT," 4th International Wireless Communications, Networking and Mobile Computing, Chongqing, 12-14 October 2008, pp. 1-4.

[5] W. Kozek and A. F. Molisch, "Nonorthogonal Pulseshapes for Multicarrier Communications in Doubly Dispersive Channels," IEEE Journal on Selected Areas in Communications, Vol. 16, No. 8, 1998, pp. 1579-1589. doi: $10.1109 / 49.730463$
[6] R. Prasad, "OFDM for Wireless Communication Systems," Artech House, Boston-London, 2004.

[7] H. Feichtinger and T. Strohmer, "Gabor Analysis and Algorithms, Theory and Applications," Birkhäuser, Berlin, 1998. doi:10.1007/978-1-4612-2016-9

[8] A. Kliks, A. Zalonis, I. Dagres, A. Polydoros and H. Bogucka, "PHY Abstraction Methods for OFDM and NOFDM Systems," Journal of Telecommunication and Information Technology, No. 3, 2009, p. 116.

[9] S. Veni and K. A. Narayanankutty, "Vison-Based Hexaganol Image Processing Using Hex-Gabor," Springer, Berlin, 2012.

[10] G. Acosta, "OFDM Simulation Using Matlab," 2000. http://www.ece.gatech.edu/research/labs/sarl/tutorials/OF DM/Tutorial_web.pdf 